\title{
Speed Performance Degradation of Electrooptic Modulator Devices by Neutrons Irradiations at High Temperature Effects
}

\author{
Ahmed Nabih Zaki Rashed \\ Electronics and Electrical Communication Engineering Department, Faculty of Electronic Engineering, \\ Menouf, Menoufia University, 32951, Egypt
}

\begin{abstract}
In the present paper, we have deeply investigated the transmission efficiency degradation of electrooptic modulator devices in thermal irradiated hard environments over wide range of the operating parameters. It is well known that the radiation-induced electrooptic modulator defects can modify the initial doping concentrations, creating generation-recombination centres and introducing trapping of carriers. Additionally, rate of the lattice defects is thermally activated and reduces for increasing irradiation temperature as a result of annealing of the damage. Both the ambient temperature and the irradiation dose possess sever effects on the electro-optical characteristics and consequently the performance characteristics of electroptic modulator devices. As well as we have deeply developed the modelling basics of electrooptic modulator devices, which may be used to analyzed the modulator quantum efficiency, dark current, modulating voltage, modulating frequency, 3-dB bandwidth, transmitted signal bandwidth, modulator quality factor, modulator sensitivity, modulator sensitivity bandwidth product, switching voltage, modulator device performance index, operating switching time and speed response of these irradiated electrooptic modulator devices after different irradiation fluences.
\end{abstract}

Keywords Radiation Damage, Switching Time, Quality Factor, Neutrons Irradiations, Switching Voltage

\section{Introduction}

Modulators and optical fibers have previously been irradiated with neutrons within thermal effects[1]. The modulators were unaffected in terms of their reflectance. The leakage current, which does not affect their performance, increased from $5 \mathrm{nA}$ to $100 \mathrm{nA}$. Of the fibers that were irradiated, pure silica core fibers were found to be more radiation hard than Ge-doped fiber. This result was expected as the induced losses in the fibres are believed to be related to the defects introduced by dopants such as germanium. The dose rates used were around two orders of magnitude greater than those expected at high irradiation doses. Therefore, taking fibre annealing into consideration, the pure silica core fibres are sufficiently resistant ton on-ionising radiation damage for high irradiation fluence use[2]. The modulators are based on III-V semiconductor electro-absorptive multi quantum well structures grown between asymmetric Fabry-Perot mirrors. The electro-absorption in the devices tested was tuned to obtain a maximum modulation depth at a wavelength of $1.54 \mu \mathrm{m}$. Reflectivity of the device is modula-

* Corresponding author:

ahmed_733@yahoo.com (Ahmed Nabih Zaki Rashed )

Published online at http://journal.sapub.org/ijoe

Copyright (C) 2011 Scientific \& Academic Publishing. All Rights Reserved ted by varying the applied electric field across the quantum wells. The devices tested here were configured as a linear array of 8 channels, with active modulator quantum well areas of $30 \mu \mathrm{m}$ diameter at $125 \mu \mathrm{m}$ pitch.

Optical communication systems are becoming the norm in communication applications. These systems have many advantages over electrical communication systems: low weight and size, mechanical simplicity, high data rates, high sensitivity and multiplexing capabilities and resistance to electromagnetic interference (EMI). Those advantages make this technology very attractive. They are preferable in radiation environments such as space borne systems, medical applications and nuclear facilities[2]. Their applications involve communication, remote sensing and monitoring. Whereas most efforts are oriented towards assessing the applicability of optical and photonic components for data transmission, far less attention is paid to the use of these components as sensors. In radiation environments, the optical sensors might be an attractive alternative to conventional measurement techniques[3]. Other applications include fiber-optic time-resolved laser-induced fluorescence for uranium and nitrate sensing, fiber optic Raman spectroscopy for waste tank monitoring and fiber dosimetry with scintillating optical fibers or induced absorption in optical fibers. Fiber-optics are envisaged for the construction of image guides or fiberscope-type viewing systems as an alternative to very 
radiation sensitive charge coupled devices (CCD) cameras to achieve visual inspection in nuclear power plants. There is a great need for optical communication for space flight usage for remote sensing and in optical communications applications. For remote sensing applications the fiber laser provides: an ultra stable compact source for laser vibrometry. Also the sensing application of photonic technologies to space including velocity sensors for gyroscope systems. The combination of fiber optic gyroscope and accelerometer measurements determine the instantaneous position and trajectory vehicle[4].

The modulators were reverse biased at -7 Volt during the irradiation in order to monitor any annealing of the leakage current which was measured and recorded every 60 seconds. Radiation damage caused the leakage current in most of the channels to increase to about $100 \mathrm{nA}$. In comparison, the photocurrent generated by the modulator under normal operating conditions is in the range of 10 to $100 \mu \mathrm{A}$ [4]. In most cases the leakage current was fully annealed in the three days after irradiation. The channels which had not annealed in this time were remeasured later and the leakage current was found to have decreased to preirradiation levels. The increases in the leakage current are thought to be associated with radiation induced charging of the polyimide layer used to isolate neighbouring multi quantum well structures[5]. This hypothesis may be confirmed by future tests on similar devices without polyimide.

In the present study, Measurements of the reflectivity of the electrooptic modulators at different bias voltages were made before and after irradiation showing no significant change. The modulators were irradiated under bias with the dark current in the devices continuously monitored during different doses after the irradiation. Increases in the dark current were observed due to radiation damage though the devices were fully annealed after irradiation fluences. Our results have suggested that the electrooptic modulator devices are sufficiently resistant to ionizing radiation though further tests are required. The transmission efficiency, sensitivity bandwidth product and device performance index were monitored throughout the irradiation for sufficient period time after irradiation.

\section{Modeling Analysis}

The point defects result in the introduction of allowed energy states within the forbidden gap of the semiconductor. This energy states lead to the following: (a) Carrier removal is the majority carrier density is reduced by the radiation fluence, (b) Mobility degradation is the mobility was found to decrease with increasing the radiation fluence[6], (c) Conductivity modulation since the carrier concentration and mobility both decrease with radiation, then the conductivity will also decrease, and (d) Minority carriers lifetime can be defined as the degradation rate in minority carrier lifetime and can be expressed as:

$$
\frac{d \tau}{d \phi}=K_{\tau}
$$

Where $\mathrm{K}_{\tau}$ is the carrier lifetime damage constant. The minority carrier lifetime $\tau$ will be reduced by[12]:

$$
\frac{1}{\tau}=\frac{1}{\tau_{0}}+\sum_{i} v_{t h} \sigma_{i} n_{i}
$$

Where $\tau_{0}$ is the pre-irradiation minority carrier lifetime, $v_{\text {th }}$ is the average thermal velocity of the minority carriers, $\mathrm{n}_{\mathrm{i}}$ and $\sigma_{i}$ are the density and capture cross section, respectively, of the recombination centers of a given type $i$, and the sum extends over the different types of radiation induced defects. As long as there is no significant overlap of the defect regions produced by the individual incident particles, the initial defect density will be proportional to the particle fluence $\varphi$ as follows:

$$
n_{i}=c_{i} \phi I
$$

Where the coefficient $c_{i}$ stands for the density of particular defects per unit fluence. Defining a damage constant $\mathrm{K}_{\tau}$ is:

$$
K_{\tau}=v_{t h} \sum_{i} c_{i} \sigma_{i}
$$

Equation 3 can then be written as the following formula[7]:

$$
\frac{\tau_{0}}{\tau}=1+\tau_{0} K_{\tau} \phi
$$

$\mathrm{K}_{\tau}$ is sensitive to many factors such as material, type of radiation, particle energy, flux, device temperature and electrical bias conditions. The ratio $\tau_{0} / \tau$ of the minority carrier lifetime before and after the irradiation can be related to the relative light output after irradiation assuming that the total current density in the junction is dominated by diffusion currents as the following expression[8]:

$$
\left(\frac{L_{0}}{L}\right)^{0.66}=\frac{\tau_{0}}{\tau}
$$

Where $\mathrm{L}_{0}$ is pre-irradiation light output and $\mathrm{L}$ is light output after irradiation. The quality factor of the EO modulator device $\mathrm{Q}$ can be expressed as the following[9]:

$$
Q=\sqrt{m_{1}}-\sqrt{m_{0}}
$$

Where $\mathrm{m}_{0}=\mathrm{m}_{\mathrm{b} 0}+\mathrm{m}_{\mathrm{d}}$ is the average number of electrons representing the 'zero' symbol, $\mathrm{m}_{1}=\mathrm{m}_{\mathrm{b} 1}+\mathrm{m}_{\mathrm{d}}$ is the average number of electrons representing the 'one' symbol, $\mathrm{m}_{\mathrm{d}}=\mathrm{i}_{\mathrm{d}}$ $\mathrm{T}_{\mathrm{s}} / \mathrm{q}$ is the average number of electrons correspond the dark current $i_{d}$ during the switching time $T_{s}$.

Where $\quad \mathrm{m}_{\mathrm{b} 0}=\lambda \eta \mathrm{P}_{0}(\mathrm{I}, \mathrm{T}, \varphi) \mathrm{T}_{\mathrm{s}} / \mathrm{hc}$

$$
\mathrm{m}_{\mathrm{bl}}=\lambda \eta \mathrm{P}_{1}(\mathrm{I}, \mathrm{T}, \varphi) \mathrm{T}_{\mathrm{s}} / \mathrm{hc}
$$

Where $\eta$ is the efficiency of EO modulator device, $h$ is the Plank's constant $\left(6.625 \times 10^{-34} \mathrm{~J} . \mathrm{Sec}\right), \mathrm{q}$ is the electron charge $\left(1.602 \times 10^{-19} \mathrm{C}\right), \mathrm{P}_{0}(\mathrm{I}, \mathrm{T}, \varphi)$ is the optical power representing the 'zero' symbol under the effects of drive current, temperature and irradiation fluence, and $\mathrm{P}_{1}(\mathrm{I}, \mathrm{T}, \varphi)$ is the optical power representing the 'one' symbol under the effects of drive current, temperature and irradiation fluence. The sensitivity (S) of the EO modulator devices can be written as[10]:

$$
S=\frac{0.65 Q \Gamma r_{33} n_{e}^{2}}{d}
$$


Where $\mathrm{Q}$ is the quality factor of the $\mathrm{EO}$ modulator device. The 3-dB electrical bandwidth, $\mathrm{f}_{3 \mathrm{~dB}}$ of an EO modulator is defined as the frequency at which the received radio frequency power drops to 0.5 of the low-frequency value or when the effective voltage on the modulator drops to 0.707 of the low-frequency value[11].

$$
B . W_{3-d B}=\frac{c}{\lambda Q}
$$

The TE-TM phase-difference modulator is realized via the tensor nature of the EO polymer and its EO effect, with the applied modulating voltage $V_{m}$, the change of the phase difference between the TM and TE modes can be as[12]:

$$
\Delta \varphi_{T E-T M} \approx-\frac{2 \pi n_{e}^{3} r_{33} \Gamma V_{m} L_{m}}{3 \lambda d}
$$

Equation (12) can be expressed in another formula in terms of modulating power as follows:

$$
\Delta \varphi_{T E-T M} \approx-\frac{2.073 n_{e}^{3} r_{33} \Gamma P(I, T, \phi) L_{m}}{\lambda d I}
$$

Where $d$ is the thickness of the EO polymer modulator in $\mathrm{cm}, \mathrm{L}_{\mathrm{m}}$ is the modulator length in $\mathrm{cm}, \mathrm{n}_{\mathrm{e}}$ is approximately the refractive index of the material based $\mathrm{EO}$ polymer modulator device, $\lambda$ is the operating signal wavelength in $\mu \mathrm{m}, r_{33}$ is the electro-optic coefficient, and $\Gamma$ is confinement factor, and is defined as the overlap integration of the modulating electrical field and the optical mode. Where the effective refractive index $n_{e}$ of polymer material based electrooptic modulator device can be expressed as[13]:

$$
n_{e}=\sqrt{\frac{S_{1} \lambda^{2}}{\lambda^{2}-S_{2}^{2}}+\frac{S_{3} \lambda^{2}}{\lambda^{2}-S_{4}^{2}}+\frac{S_{5} \lambda^{2}}{\lambda^{2}-S_{6}^{2}}}
$$

The set of parameters of empirical equation coefficients of polymer material are recast as the following[14]: $\mathrm{S}_{1}=0.4963$, $\mathrm{S}_{2}=0.0718\left(\mathrm{~T} / \mathrm{T}_{0}\right)^{2}, \mathrm{~S}_{3}=0.6965, \mathrm{~S}_{4}=0.1174\left(\mathrm{~T} / \mathrm{T}_{0}\right)^{2}, \mathrm{~S}_{5}=0.3223$, and $\mathrm{S}_{6}=9.237\left(\mathrm{~T} / \mathrm{T}_{0}\right)^{2}$. Where $\mathrm{T}$ is the ambient temperature, and $\mathrm{T}_{0}$ is the room temperature. The first and second differentiation of empirical equation with respect to operating wavelength $\lambda$ as yields in Ref.[14]. The transmission of a Mach-Zehnder (MZ) EO polymer modulator is given by[15]:

$$
T(\lambda)=0.5\left[1+\exp \left(\frac{\pi P(I, T, \phi)}{I V_{\pi}}+\varphi_{B}\right)\right]
$$
by:

Where the phase bias of EO polymer modulator is given

$$
\varphi_{B}=\varphi_{\Delta}+\varphi_{v}=\frac{2 \pi \Delta n_{e}}{\lambda}+\frac{\pi V_{B}}{V_{\pi}}
$$

Where $\Delta \mathrm{n}_{\mathrm{e}}$ is the effective relative refractive-index change, $\mathrm{V}_{\mathrm{B}}$ is the applied bias voltage in Volt, and $\mathrm{V}_{\pi}$ is the switching voltage or the voltage required to change the output light intensity from its maximum value to its minimum value can be expressed as the following[16]:

$$
V_{\pi}=\frac{\lambda I^{2} \rho}{2 \Gamma n_{e}^{3}(\lambda) r_{33} P(I, T, \phi) W}
$$

Where $\mathrm{W}$ is the modulator thickness in $\mu \mathrm{m}, \rho$ is the specific resistivity of the material based EO modulator devices, and $I$ is the drive current in $\mathrm{mA}$. Under the perfect velocity matching condition[17], achievable modulation bandwidth $\mathrm{f}_{\mathrm{m}}$ can be expressed as the $\mathrm{f}$ :

$$
f_{m}=\frac{6.84 \rho I^{2}}{\alpha P(I, T, \phi) d W}, \mathrm{GHz}
$$

Where $\alpha$ the power absorption coefficient within $0.1-0.5$ $\mathrm{dB} / \mu \mathrm{m}, \mathrm{d}$ is the thickness of the modulator in $\mu \mathrm{m}$. Moreover the EO modulator device performance index (DPI) can be expressed as follows[18]:

$$
D P I=\frac{f_{m}}{V_{\pi}}, G H z / \text { Volt }
$$

The power forward current voltage curves of different types of EO modulator devices were given in[18], with remarkable nonlinearly while in[19] it depicted in linear fashion. Based on the data of[20], the following nonlinear thermal relations for the set of the selected device were carried out:

$$
\begin{aligned}
& P(I, T)=p_{0}+p_{1} I+p_{2} I^{2}, \text { mWatt } \\
& V(I, T)=v_{0}(T)+v_{1} I+v_{2} I^{2}, \text { Volt }
\end{aligned}
$$

Where the set of parameters $\left\{\mathrm{p}_{0}, \mathrm{p}_{1}, \mathrm{p}_{2}\right.$, and $\left.\mathrm{v}_{\mathrm{o}}\right\}$ are polynomial functions of $\mathrm{T}$.

$$
\begin{aligned}
p_{0} & =0.721-0.0064 T+0.03465 T^{2} \\
p_{1} & =0.5-0.0543 T+0.0321 T^{2} \\
p_{2} & =-7.324-0.00322 T+0.00662 T^{2} \\
v_{0} & =0.521-0.02315(T-300)
\end{aligned}
$$

Where the set of the coefficients of $\mathrm{v}_{1}=1.876$, and $\mathrm{v}_{2}=-0.5407$. The power forward current and bias voltage of modulator under the effects of irradiation can be expressed in the following formulas[21]:

$$
\begin{aligned}
& P(I, T, \phi)=P(I, T) F_{p}(\phi) \\
& V(I, T, \phi)=V(I, T) F_{v}(\phi)
\end{aligned}
$$

Where both $\mathrm{F}_{\mathrm{p}}(\varphi)$ and $\mathrm{F}_{\mathrm{v}}(\varphi)$ are functions of the irradiation fluence $\varphi$, can be expressed as follows[22]:

$$
\begin{gathered}
F_{p}(\phi)=1+\alpha_{1} \phi+\alpha_{2} \phi^{2} \\
F_{v}(\phi)=1+\beta_{1} \phi+\beta_{2} \phi^{2}
\end{gathered}
$$

Where the set of the coefficients of $\alpha_{1}=0.0025, \alpha_{2}=-0.043$, $\beta_{1}=0.623$, and $\beta_{2}=0.03224$.

\section{Simulation Results and Performance Analysis}

We have investigated the transmission efficiency degradation of electrooptic modulator devices in thermal irradiated hard environments under the set of the wide range of the affecting and operating parameters are listed as follows: Confinement factor $\Gamma=0.8-0.95$, Specific resistivity $\rho=$ $2.66 \times 10^{-6} \Omega . \mu \mathrm{m}$, Electro-optic coefficient $r_{33}=300 \mathrm{pm} /$ Volt, Relative refractive index difference $\Delta \mathrm{n}_{\mathrm{e}}=0.01$, Bias voltage $\mathrm{V}_{\mathrm{B}}=2$ Volt, Modulator thickness $\mathrm{d}=0.02 \mu \mathrm{m}$, Dark current $\mathrm{i}_{\mathrm{d}}$ $=0.01 \mu \mathrm{A}$, Switching time $\mathrm{T}_{\mathrm{s}}=0.8 \mathrm{msec}$, Speed of light $\mathrm{c}=3$ $\mathrm{x} 10^{10} \mathrm{~cm} / \mathrm{sec}$, Modulator length $\mathrm{L}_{\mathrm{m}}=0.2 \mu \mathrm{m}$, Modulator width $\mathrm{W}=0.1 \mu \mathrm{m}$, Room temperature $\mathrm{T}_{0}=300 \mathrm{~K}$, Ambient temperature $\mathrm{T}=300-340 \mathrm{~K}$, Operating signal wavelength $\lambda=$ $1.55 \mu \mathrm{m}$, Electron charge $\mathrm{q}=1.6 \times 10^{-19} \mathrm{C}$, Quantum effi- 
ciency $\eta=90 \%$, applied drive current $\mathrm{I}=2 \mathrm{~mA}$, irradiation fluence or dose $\varphi=2 \times 10^{14}-50 \times 10^{14} \mathrm{n} / \mathrm{cm}^{2}$. Based on the model equations analysis, assumed set of the operating parameters as listed previously, and based on the series of the Figs. (1-7), the following facts are assured:

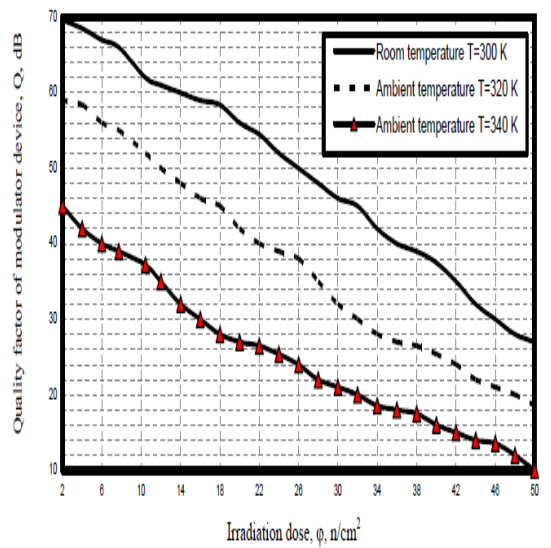

Figure 1. Variations of the quality facter of electrooptic modulator against irradiation dose at the assumed set of parameters

i. As shown in Fig. 1 has assured that as both irradiation doses and ambient temperature increase, this leads to decrease in the quality factor of modulator devices.

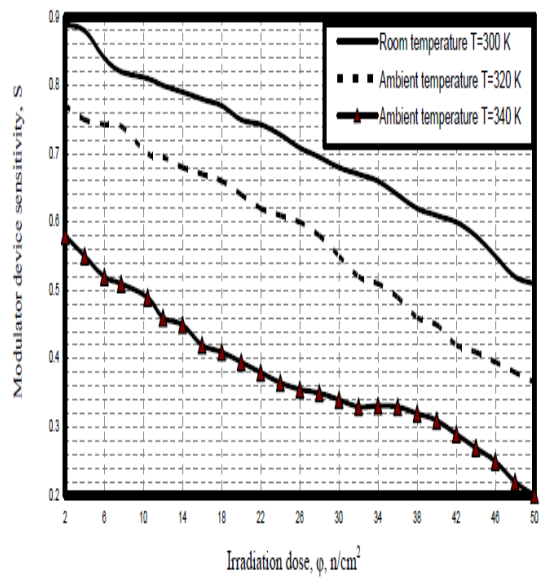

Figure 2. Variations of the electrooptic modulator device sensitivity against irradiation dose at the assumed set of parameters

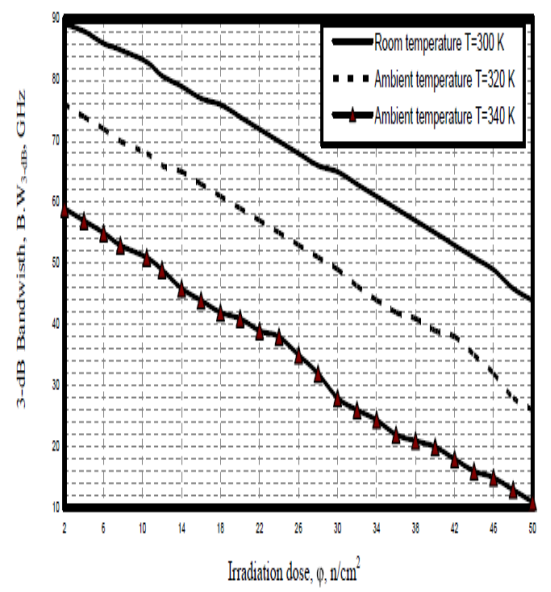

Figure 3. Variations of 3-dB signal transmitted bandwidth against irradiation dose at the assumed set of parameters ii. Fig. 2 has proved that as both irradiation doses and ambient temperature increase, this results in decreasing of modulator device sensitivity.

iii. As shown in Fig. 3 has demonstrated that as both irradiation doses and ambient temperature increase, this results in decreasing of $3-\mathrm{dB}$ bandwidth of the received signal within electrooptic modulator devices.

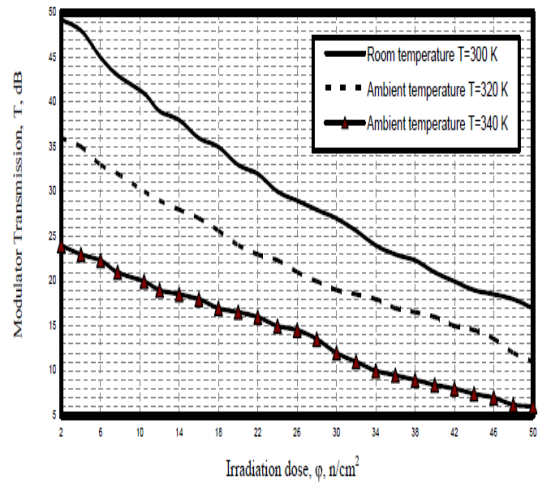

Figure 4. Variations of modulator device signal transmission against irradiation dose at the assumed set of parameters

iv. Fig. 4 has indicated that that as both irradiation doses and ambient temperature increase, this leads to decrease in modulator device transmission that affect directly in the modulator speed performance.

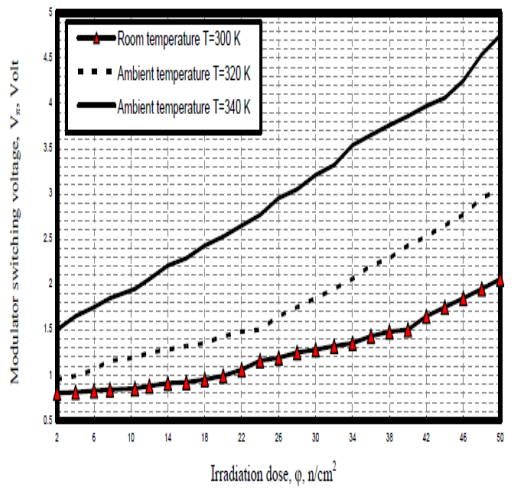

Figure 5. Variations of modulator device switching voltage against irradiation dose at the assumed set of parameters

v. As shown in Fig. 5 has assured that $\mathrm{s}$ both irradiation doses and ambient temperature increase, this results in increasing of modulator device switching voltage.

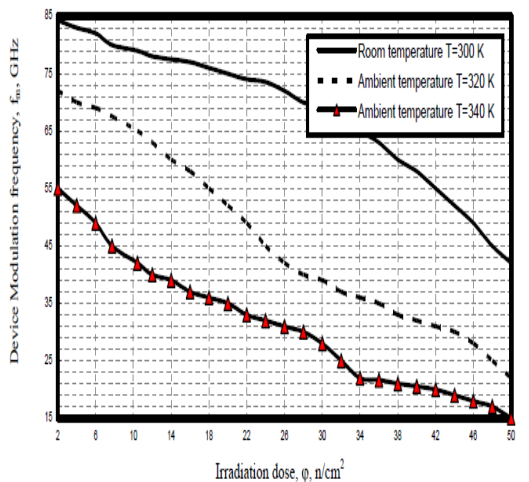

Figure 6. Variations of modulator device modulation frequency against irradiation dose at the assumed set of parameters 
vi. Fig. 6 has indicated that that as both irradiation doses and ambient temperature increase, this leads to decrease in modulation device frequency that affect directly in the modulator speed transmission efficiency.

vii. As shown in Fig. 7 has demonstrated that as both ambient temperatures and irradiation doses increase, this results in decreasing of modulator device performance index that has the great bad effects on modulator speed performance and transmission efficiency.

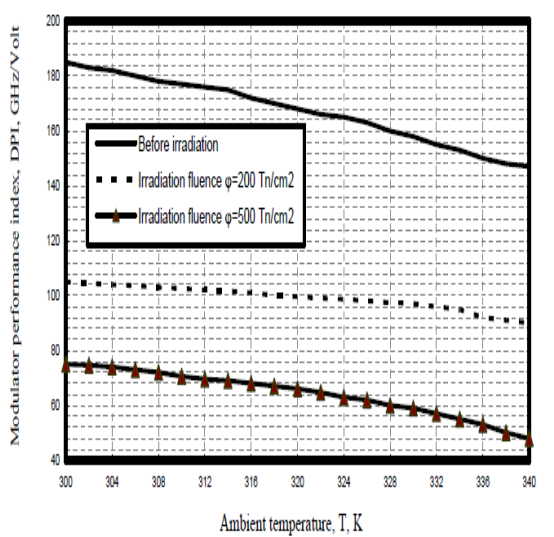

Figure 7. Variations of modulator device performance index against ambient temperature at the assumed set of parameters

\section{Conclusions}

In a summary, we have been deeply investigated the speed performance degradation of electrooptic modulator devices under high temperature variations and different irradiation doses environment. It is theoretically found that the increase of both irradiation doses and ambient temperature variations, the decreased modulator device quality factor, modulator device sensitivity, transmitted signal bandwidth, modulator transmission, device modulation frequency, and the modulator device performance index. It is also evident that the increased ambient temperature and irradiation doses, this leads to the increased modulator switching or driving voltage. Thus the increased thermal irradiated fields has the great harmful effects on the modulator speed performance and transmission efficiency. To the best of our knowledge, high thermal irradiated environments study on the highest driving voltage and operating device parameters of electrooptic modulators has been performed particularly operating at 1.55 mm wavelength.

\section{REFERENCES}

[1] S. M. Khanna, D. Estan, L. S. Erhardt, A. Houdayer, C. Carlone, A. Ionascut-Nedelcescu, S. R. Messenger, R. J. Walters, G. P. Summers, J. H. Warner, and I. Jun, "Proton energy dependence of the light output in gallium nitride light emitting diodes," IEEE Trans. Nucl. Sci., vol. 51, pp. 2729-2735, Oct. 2004

[2] A. Kalavagunta, M. Neifeld, H. Barnby, and R. Schrimpf,
"Impact of proton irradiation on a grating demultiplexer," IEEE Trans. Nucl. Sci, vol. 51, pp. 3595-3602, Dec. 2004

[3] J. H. Warner, R. J. Walters, S. R. Messenger, G. P. Summers, S. M. Khanna, D. Estan, L. S. Erhardt, and A. Houdayer, "High energy proton irradiation effects in GaAs devices," IEEE Trans. Nucl. Sci., vol. 51, pp. 2887-2895, Oct. 2004

[4] M. Pattabi, S. Krishnan, and G. Sanjeev, "Studies on the temperature dependence of $\mathrm{I}-\mathrm{V}$ and $\mathrm{C}-\mathrm{V}$ characteristics of electron irradiated silicon photo-detectors," J. Solar Energy Materials \& Solar Cells 91, pp.1521-1524, 2007

[5] S. Krishnan, G. Sanjeev, and M. Pattabi, " $8 \mathrm{MeV}$ electron irradiation effects in silicon photo-detectors," Nucl. Instr. and Meth. in Physics Research B 264, pp. 79-82, 2007

[6] A. Simon, G. Kalinka, M. Jaks, Z. Pastuovic, M. Nova and A.Z. Kiss, "Investigation of radiation damage in a Si PIN photodiode for particle detector," Nucl. Instr. and Meth. in Physics Research B 260, pp. 304-308, 2007

[7] M. Van Uffelen, Ivan Genchev and F. Berghmans, "Reliability study of photodiodes for their potential use in future fusion reactor environments," SPIE Proceeding 5465, pp. 92-102, 2004

[8] K. Gill, S. Dris, R. Grabit, R. Macias, E. Noah, J. Troska and F. Vasey, "Radiation hardness assurance and reliability testing of InGaAs photodiodes for optical control links in the CMS experiment," IEEE Trans. On Nucl. Sci., Vol. 5, No. 2, pp. 1480-1487, 2005

[9] Abd El-Naser A. Mohammed, Ahmed Nabih Zaki Rashed, and Mahmoud M. Eid, "Important Role of Optical Add Drop Multiplexers (OADMs) With Different Multiplexing Techniques in Optical Communication Networks," International Journal of Computing, Vol. 9, No. 2, pp. 152-164, 2010

[10] M. McPherson, "Infrared photoconduction in radiation-damaged silicon diodes," J. Opt. A: Pure Appl. Opt. 7, pp. S325-S330, 2005

[11] Shinobu Onoda, Toshio Hirao, Hisayoshi Itoh and Tsuyoshi Okamoto, "Evaluation of Transient Current in Si PIN photodiode Induced by High-Energy Charged Particles," Proc. Sch. Eng. Sch. Inf. Sci. Tokai Univ. 31, pp. 1-4, 2006

[12] B. Danilchenko, A. Budnyk, L. Shpinar, D. Poplavskyy, S.E. Zelensky, K.W.J. Barnham and N.J. Ekins, "1 MeV electron irradiation influence on GaAs solar cell performance," J. Solar Energy Materials and Solar Cells 92, pp. 1336- 1340, 2008

[13] Abd El-Naser A. Mohammed, Abd El-Fattah A. Saad, and Ahmed Nabih Zaki Rashed, "Characteristics of the Fabrication Materials Based Arrayed Waveguide Grating (AWG) in Passive Optical Networks (PONs)," International Journal of Material Sciences Research, Vol. 1, No. 6, pp. 89-97, June 2009

[14] Abd El-Naser A. Mohammed, Gaber E. S. M. El-Abyad, Abd El-Fattah A. Saad, and Ahmed Nabih Zaki Rashed, "Low Loss A thermal Arrayed Waveguide Grating (AWG) Module for Passive and Active Optical Network Applications," International Journal of Communication Networks and Information Security (IJCNIS), Vol. 1, No. 2, pp. 27-34, August 2009

[15] H. Ohyama, K. Takakura, K. Hayama, S. Kuboyama, Y. Deguchi, and S. Matsuda, E. Simoen and C. Claeys, "Damage coefficient in high-temperature particle and $\gamma$-irradiated silicon $\mathrm{p}-\mathrm{i}-\mathrm{n}$ diodes,” J. Appl. Phys. Lett., Vol. 82, No. 2, pp. 296-298, 2003

[16] M. A. Cappelletti, A.P. C'edola1 and E.L. Peltzer y. Blanc'a, "Simulation of silicon PIN photodiodes for use in 
space-radiation environments," Semicond. Sci. Technol., Vol. 23, No. 2, pp. 7-13, 2008

[17] K. Konno, O. Matsushima, D. Navarro, and M. Miura-Mattausch, "High frequency response of p-i-n photodiodes analyzed by an analytical model in Fourier space," J. Appl. Phys., Vol. 96, No. 7, pp. 3839-3844, 2004

[18] S. R. Messenger, E. A. Burke, M. A. Xapsos, G. P. Summers, R. J.Walters, I. Jun, and T. M. Jordan, "NIEL for heavy ions: An analytical approach,” IEEE Trans. Nucl. Sci., vol. 50, pp. 1919-1923, 2003

[19] I. Jun, M. A. Xapsos, S. R. Messenger, E. A. Burke, R. J. Walters, G. P. Summers, and T. Jordan, "Proton nonionizing energy loss (NIEL) for device applications," IEEE Trans. Nucl. Sci., vol. 50, pp. 1924-1928, 2003
[20] Sh.M. Eladl, "Modeling of photons trapping effect on the performance of HPT-LED Optoelectronic Integrated Device (OEID)," Semiconductor Physics, Quantum Electronics \& Optoelectronics Journal, Vol. 12, No. 3, pp. 255-259, 2009

[21] Abd El-Naser A. Mohammed, Abd El-Fattah A. Saad, and Ahmed Nabih Zaki Rashed, "Study of the Thermal and Spectral Sensitivities of Organic-Inorganic Fabrication Materials Based Arrayed Waveguide Grating for Passive Optical Network Applications," Journal of Engineering and Technology Research, Vol. 1, No. 5, pp. 81-90, August 2009

[22] M. V. Raghavendra, and P. L. H. Vara Prasad, "Estimation of Optical Link Length for Multi Haul Applications," International Journal of Engineering Science and Technology, Vol. 2, No. 6, pp. 1485-1491, 2010 